\title{
SONOGRAPHIC ELASTOGRAPHY IN ORAL AND MAXILLOFACIAL IMAGING- A REVIEW
}

\author{
Wagisha Barbi', Archana Sudheer², Nimmi Singh³, Kumar Anand ${ }^{4}$
}

${ }_{1}^{1}$ Postgraduate Student, Department of Oral Medicine \& Radiology, Buddha Institute of Dental Sciences \& Hospital, Patna.

2 Professor \& HOD, Department of Oral Medicine \& Radiology, Buddha Institute of Dental Sciences \& Hospital, Patna.

${ }^{3}$ Reader, Department of Oral Medicine \& Radiology, Buddha Institute of Dental Sciences \& Hospital, Patna.

${ }^{4}$ Senior Lecturer, Department of Oral Medicine \& Radiology, Buddha Institute of Dental Sciences \& Hospital, Patna.

\section{ABSTRACT}

\section{BACKGROUND}

Sonographic elastography is a new sensitive ultrasonic imaging technique for measurement of the tissue thickness. In sonographic elastography, information of tissue strain or shear wave is imaged when the vibration energy is given to target tissue. There are different types of sonographic elastography tests like strain elastography, acoustic radiation force impulse imaging technique and shear elastography. There is a different possibility of application of sonographic elastography to the oral and maxillofacial structures such as salivary gland malignancies, cervical lymph nodes and in muscles especially masseter. This article aims to discuss the advances in recent application of sonographic elastography.

\section{KEYWORDS}

Elastography, Sonographic Elastography, Salivary Gland, Lymph Node, Masseter Muscles.

HOW TO CITE THIS ARTICLE: Barbi W, Sudheer A, Singh N, et al. Sonographic elastography in oral and maxillofacial imaging- A review. J. Evolution Med. Dent. Sci. 2017;6(84):5884-5886, DOI: 10.14260/jemds/2017/1278

\section{BACKGROUND}

Elastography is also known as 'palpation imaging'. Ultrasonic elastography was first described in 1987 by Krouskop et al. It is an imaging technique where elasticity of tissue is determined non-invasively to differentiate between normal and diseased tissue. Thus, the basis of elastography is analogous to manual palpation. Previously virtual palpation or manual palpation was used to measure the stiffness. Palpation continues to be of great importance and value in modern medicine, which is widely used by doctors and even it is used as a technique for self-examination. However, this 'palpation' technique has its limitations, like it is limited to few accessible organs only, and interpretation of the information sensed by the fingers is highly subjective. Elastography is a newly developed dynamic technique that uses ultrasound to provide an estimation of tissue stiffness by measuring the degree of distortion under the application of external force. There are different types of elastography like Ultrasound Elastography and Magnetic Resonance Elastography among which Ultrasound Elastography is a widely used technique. Abnormal tissue will appear more stiffer than normal tissue. Stiff tissues deform less and exhibit less strain than compliant tissues in response to the same applied force.

\section{Principle of Elastography}

Elastography is a promising imaging tool because the elastic modulus of tissues measured by this technique provides the most broad-banded properties compared with other quantitative values measured by computed tomography

'Financial or Other Competing Interest': None.

Submission 14-09-2017, Peer Review 06-10-2017,

Acceptance 14-10-2017, Published 19-10-2017.

Corresponding Author:

Dr. Wagisha Barbi,

C/o. Dr. Prem Kumar

Type- 5, Block-B, AIIMS Residential Campus,

Phulwarisharif, Khagaul, Patna-801105.

E-mail: wagishabarbie@gmail.com

DOI: $10.14260 /$ jemds/2017/1278 (attenuation value), magnetic resonance (MR) imaging (T1 relaxation time) and conventional ultrasonography (bulk modulus). ${ }^{1}$ So the elastic modulus can be defined as the slope of the stress-strain curve during elastic deformation. The order of magnitude of the elastic modulus is approximately five times larger than that for other imaging modalities, there by stating that the use of the elastic modulus can maximise the discrimination between different tissues or between normal tissue and lesions up to five times the normal.

So the principle states that a stiffer object has a higher elastic modulus. So, softer parts of tissues deform easily than the harder tissue under same compression, thus allowing an objective deformation of tissue consistency. ${ }^{2}$ There are three basic steps in elastography - a) excitation: transmission of stress in a tissue (mechanical, vibration, shear); b) acquisition: - recording the signal induced by the tissue deformation due to stress; c) Analysis - analysis of tissue strain induced by the propagation of the stress. Figure 1 .

\section{Types of Elastography}

1. Ultrasound Elastography.

- Real Time Elastography.

2. Strain Elastography.

3. Strain - Stress Elastography (Semi-quantitative Elastography).

- Quantitative Elastography.

a. Transient Elastography.

b. ARFI Elastography.

c. Shear Wave Elastography.

d. MR Elastography.

\section{Technique of U/S Elastography}

- To acquire two ultrasonic images (before and after tissue compression by the probe) and track tissue displacement by assessing the propagation of the imaging beam.

- A dedicated software that is able to provide an accurate measurement of tissue distortion is used. 
- $\quad \mathrm{U} / \mathrm{S}$ elastogram is displayed over the B-mode image in a colour scale that ranges from red, for components with greatest elastic strain (i.e. softest components), to blue for those with no strain (i.e. hardest components).

- U/S elastographic image is matched with an elasticity colour scale.

- It is important that the level of pressure is maintained constant throughout the examination.

- Easy to perform and requires no more than 3-5 min. of additional examination time. ${ }^{2}$

\section{Colour Coding In Elastography}

Elastogram is an image that shows different shades of colour corresponding to different degree of stiffness resulting from compression. Images obtained by elastography are compared before and after compression. Elasticity varies in different pathological conditions like inflammation and malignancy. Elasticity score is indicated by diverse colours. Colour ranges from red to blue. Red colour indicates soft tissue, green colour indicates tissue with intermediate stiffness and blue colour represents hard tissue. ${ }^{3}$ Figure 2.

\section{Clinical Applications of Sonographic Elastography In Oral \& Maxillofacial Region}

\section{Salivary Glands}

Sonography is the first choice of imaging modality in evaluation of salivary neoplasm. ${ }^{4}$

Tissue elasticity in salivary glands probably reflects a combination of factors, including the quality and quantity of salivary glandular tissue, other components like fatty tissue or fibrosis which may be increased in irradiated glands. ${ }^{5}$ The stiffness of the malignant tumours are always higher than the benign neoplasm, which states that stiffness of pleomorphic adenoma is higher than that of Warthin tumour.

\section{Cervical Lymph Nodes}

The lymph node staging plays an important role in patients with head and neck cancer. ${ }^{6}$ Information regarding lymph node staging and localisation of metastatic lymph node in a patient with head and neck cancer is important and mandatory for the choice of therapy. Metastatic LN delineated by Ultrasound is an increased size of lymph node, a more round shape and cystic degeneration, keratinisation and tumour necrosis giving rise to a heterogeneity. Round shape is usually considered more suspicious of metastasis than oval and flat shape.

Four-point elastography scale is most frequently used for detecting malignant lymph nodes Asteria et al. Figure 3

Score 1-2 - demonstrates benign lymph node (LN).

Score 3-4 - demonstrates malignant lymph node (LN).

Strain elastography technique helps to differentiate reactive \& metastatic LN.

Ragoo et al gave Five-point scoring system as follows: Figure 4

Score 1-2 - demonstrates benign lymph node.

Score 3-4 - demonstrates malignant lymph node.

Score 5 - indicates metastatic lymph node.

\section{Masseter Muscles}

Sonographic elastography is a feasible additional imaging tool for knowing muscle stiffness in maxillofacial region. Muscle oedema may lead to muscle fatigue and pain in the muscles. Sustained contraction of the muscles can produce high intramuscular pressure which can impede local muscle blood flow. Blood vessel compression can lead to hypoperfusion, hypoxia which in turn causes pain.

In masseter muscle, sonographic elastography has a possibility for exploring the cause of muscle pain, for understanding the state of muscle, selecting therapeutic methods and evaluating therapeutic effect.

In patients with myofascial pain, the ratio of soft and hard areas are low but after exercise in elastography imaging the soft to hard areas ratio is increased immediately. Figure 5.

\section{Other Applications}

Ultrasonic elastography is an effective diagnostic tool to confirm benign and malignant lesion without ultrasoundguided biopsy, which is an invasive, painful process and can lead to infection.

It is also an effective tool to confirm abscess formation. Large simple cyst shows no elasticity within the lesion and hence black on the elastogram.

It is found very useful in the detection of tongue carcinoma. It could help in even estimating the depth of tumour invasion because proximity of tumour to blood vessels and lymphatics, enhances tumour's ability to metastasise which is determined by increased depth of invasion.

In oral submucous fibrosis, there is decreased blood flow, increased echogenicity and loss of subcutaneous fat which lead to fibrous bands which in turn leads to increase muscles stiffness.

In Temporomandibular disorder, masseter muscles' elasticity showed a significant difference between the elasticity ratio of symptomatic and asymptomatic side of the patient. $^{7}$

The therapeutic ultrasonic elastography thermal effects can occur with elevation of tissue temperature which increases the pain threshold and a break in pain cycle which leads in reduction of muscle spasm and increased extensibility of collagen fibres.

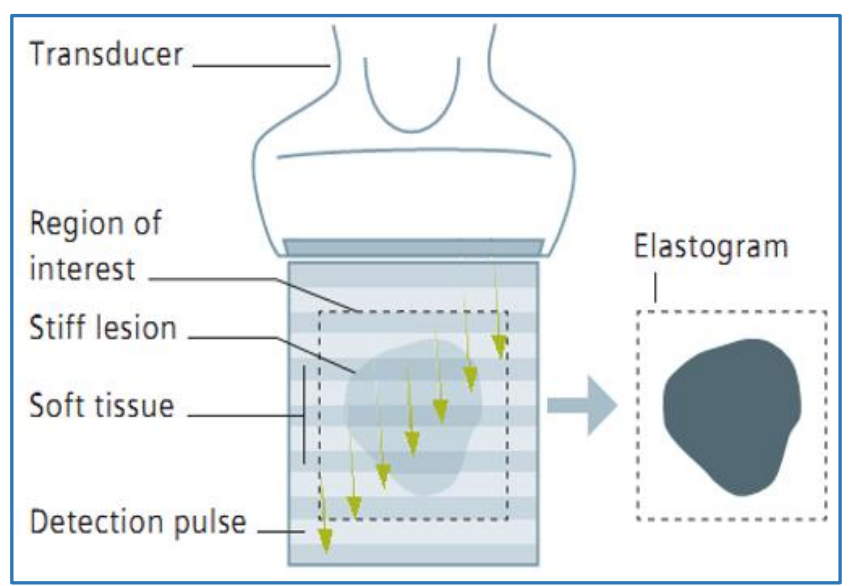

Figure 1. Principle of Elastography 


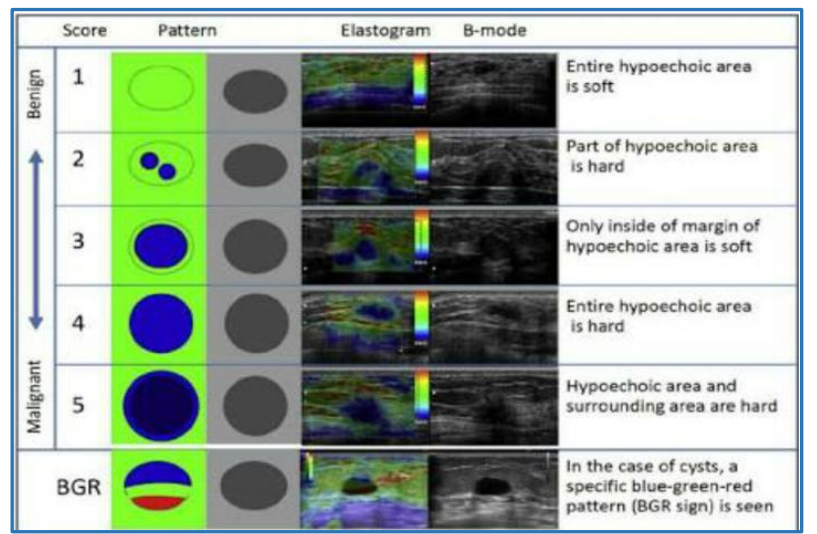

Figure 2. Elastogram Showing Colour Coding

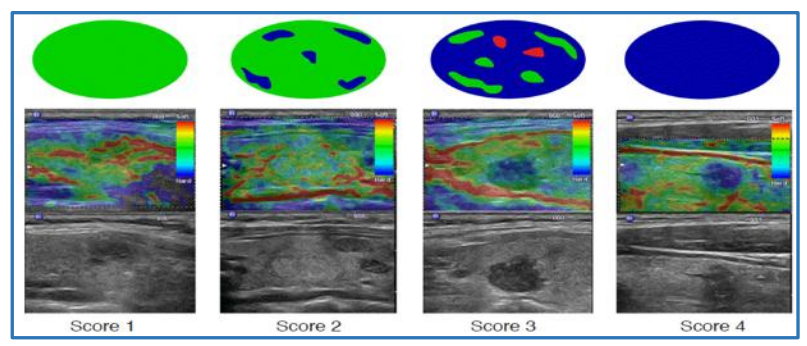

Figure 3. Four-point Elastography Scale for Detecting Malignant Lymph Nodes by Asteria et al

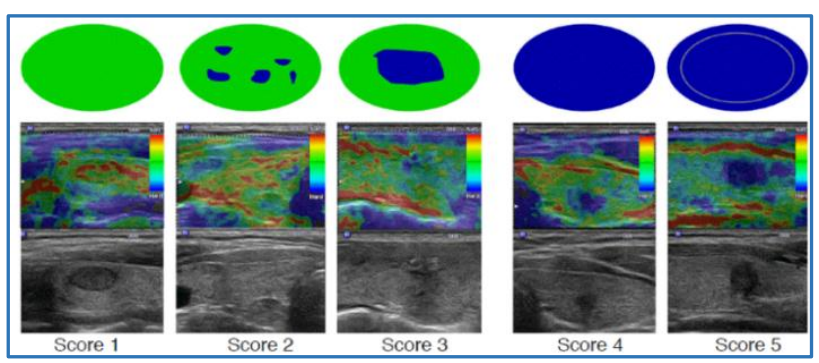

Figure 4. Five-point Scoring System by Ragoo et al

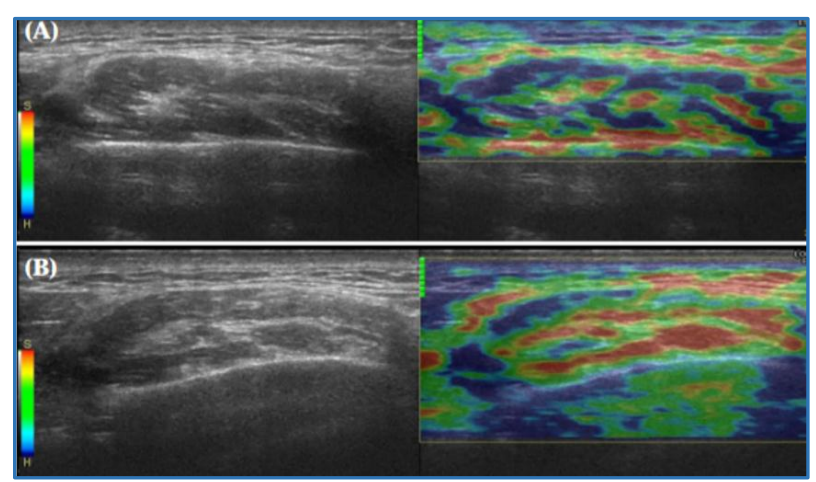

Figure 5. Strain Sonography before and after Massage Treatment in a 53-year-old Man with Myofascial Pain, A) Before Treatment, B) After Treatment. After Exercise, Elasticity Ratio is Decreased

\section{CONCLUSION}

Ultrasonic elastography is a promising tool for diagnosing benign and malignant pathologies. In general, it has its wide range of application in investigating disease in liver, breast, thyroid and prostate cancer. Now it is finding its place in head and neck imaging as in salivary gland tumours, cervical lymph nodes and muscles of mastication. Research about the application and significance of ultrasonic elastography can substantiate the importance of this new diagnostic tool in dental field.

\section{REFERENCES}

[1] Jeong WK, Lim HK, Lee HK, et al. Principles and clinical application of ultrasound elastography for diffuse liver disease. Ultrasonography 2014;33(3):149-60.

[2] Bhargava S, Bhargava SK, Sharma S, et al. Elastography: a new imaging technique and its application. JIMSA 2013;26(1):25-30.

[3] Garge VN, Waikul KV, Kadam VJ. Elastography: techniques and applications. World Journal of Pharmacy And Pharmaceutical Sciences 2016;5(3):359-68.

[4] Ariji Y, Nakayama M, Nishiyama W, et al. Application of sonographic elastography to the oral and maxillofacial region. J Radiol Radiat Ther 2014;2(2):1049.

[5] Kaluzny J, Kopec T, Parulska E, et al. Shear wave elastography: a new noninvasive tool to assess the intensity of fibrosis of irradiated salivary glands in head and neck cancer patients. BioMed Research International Article ID 157809, 2014;2014:6.

[6] Hayashi T. Application of ultrasonography in dentistry. Japanese Dental Science Review 2012;48(1):5-13.

[7] Ariji Y, Gotoh A, Hiraiwa Y, et al. Sonographic elastography for evaluation of masseter muscle hardness. Oral Radiol 2013;29(1):64-9. 